\title{
$Z Z$ production in gluon fusion at NLO matched to parton shower
}

\author{
Simone Alioli, ${ }^{*}$ Fabrizio Caola, ${ }^{\dagger}$ and Gionata Luisoni ${ }^{\ddagger}$ \\ CERN Theory Division, CH-1211 Geneva 23, Switzerland \\ Raoul Röntsch ${ }^{\S}$ \\ Institute for Theoretical Particle Physics, KIT, D-76128 Karlsruhe, Germany
}

(Received 10 October 2016; published 28 February 2017)

\begin{abstract}
We present a calculation of the next-to-leading order QCD corrections to the hadroproduction process $g g \rightarrow Z Z \rightarrow e^{+} e^{-} \mu^{+} \mu^{-}$, matched to the parton shower in the POWHEG framework. We take advantage of the POWHEG BOX tool for the implementation and rely on PYTHIA8 for the showering and hadronization stages. We fully include $\gamma^{*} / Z$ interference effects, while also covering the single-resonant region. For this phenomenological study we focus on four lepton production as a signal process, neglecting all quark mass effects as well as the Higgs-mediated contributions, which are known to be subdominant in this case. We provide predictions from our simulations for the $13 \mathrm{TeV}$ LHC Run II setup, including realistic experimental cuts.
\end{abstract}

DOI: 10.1103/PhysRevD.95.034042

\section{INTRODUCTION}

During the Run I of the Large Hadron Collider (LHC), the production of a pair of vector bosons was one of the processes studied in greatest detail [1-6]. The Higgs boson was initially discovered through its decay into dibosons, and this decay channel continues to be important for measuring the properties of the Higgs [7-10]. First LHC Run II data at $13 \mathrm{TeV}$ recently became available from both ATLAS [11] and CMS [5]. Further LHC results with higher statistics will enable a program of precision Higgs measurements, including its coupling to vector bosons through its decay to $Z Z$ or $W^{+} W^{-}$. Additionally, diboson production is a benchmark process for precision tests of the Standard Model (SM), while also providing constraints on anomalous gauge bosons couplings [12-14]. Without any direct sign of new physics, such indirect searches become increasingly relevant, and the need for high precision becomes more important.

An essential requirement of such a program is the availability of theoretical predictions for both signal and backgrounds which match the experimental precision. In the case of diboson, the lowest order production mechanism is quark-antiquark annihilation. The next-to-next-toleading order (NNLO) corrections to these processes have been computed [15-24], and have been matched to resummation of the transverse momentum of the diboson

\footnotetext{
*simone.alioli@cern.ch

†fabrizio.caola@cern.ch

gionata.luisoni@cern.ch

§raoul.roentsch@kit.edu
}

Published by the American Physical Society under the terms of the Creative Commons Attribution 3.0 License. Further distribution of this work must maintain attribution to the author(s) and the published article's title, journal citation, and DOI.
[25] and of the hardest jet [26]. Included in these corrections is the contribution from gluon-initiated production, which proceeds through a quark loop since the gluons do not couple directly to electroweak gauge bosons. Thus the leading order (LO) contribution to the $g g$ channel is given by a one-loop amplitude and first enters the overall diboson production rate at $\mathcal{O}\left(\alpha_{s}^{2}\right)$, i.e. at NNLO. It has been known for some time that these contributions increase the cross section by approximately $5 \%-15 \%$, enhanced by the large gluonic flux [27-29]. Furthermore, since these contributions have LO-like scale uncertainty, they are responsible for the majority of the residual scale uncertainty at NNLO.

The NLO corrections for gluon-induced diboson production were recently computed both for $Z Z$ [30] and for $W^{+} W^{-}[31] .{ }^{1}$ There it was shown that the next-to-leading order (NLO) corrections further enhance the production rate, resulting in an overall increase of the predictions for $Z Z$ production at the level of $\sim 5 \%$. This exceeds the scale variation uncertainty of the NNLO computation, making its inclusion important for precision phenomenology.

In order to obtain accurate predictions with generic fiducial cuts, the implementation of gluon-initiated diboson processes in a parton shower framework is highly desirable. In this paper, we present results for the NLO QCD corrections to $Z Z$ production in the gluon fusion channel matched to parton shower within the POWHEG framework. We also include contributions from topologies with a single $\gamma^{*} / Z$ resonance. In this channel, such single-resonant topologies only start to contribute at NLO. We do not consider Higgs-mediated contributions and neglect quarkmass effects throughout. As such, our study represents a first step towards a complete matching of NLO gluoninduced diboson production to parton shower.

\footnotetext{
${ }^{1}$ The interference with the Higgs-boson production channel was also recently computed in Refs. [32,33].
} 
The remainder of the paper is organized as follows. First we present the computational setup. Then we present our results, first applying a generic set of cuts, and then using realistic experimental cuts. Finally, we conclude and give a brief outlook.

\section{COMPUTATIONAL SETUP}

In this study we focus on the $g g \rightarrow Z Z \rightarrow e^{+} e^{-} \mu^{+} \mu^{-}$ channel as a signal process. We do not include Higgsmediated contributions, which are mostly relevant around the Higgs peak and at very high invariant masses (see e.g. Refs. [33,34] and [32]). The fixed-order computation is performed using the same strategy presented in Ref. [30], which we recapitulate here briefly.

We consider $Z Z$ production via gluon fusion through a loop of massless quarks only. At leading order there are two types of loop-induced diagrams: boxes [an example is shown on the left in Fig. 1(a)] and triangles [right in Fig. 1(a)]. The contribution from the latter cancels within a massless quark family at all orders. Since we are considering five light flavors and neglecting contributions from the massive top quark, for which the two-loop diagrams are not known, the cancellation of these contributions between top and bottom quarks is broken, giving rise to an anomaly. In order to avoid this we neglect triangle diagrams at all orders, and work with five active massless flavors, see Ref. [30] for further details. At LO, the neglected contributions affect the total $g g$-initiated cross section at the level of $1 \%$, and restrict the validity of the predictions to invariant masses of the four lepton system, $m_{4 \ell}$, smaller than roughly twice the top-quark mass [34,35]. As a consequence, in our study we only show results in this kinematic region.



(a)

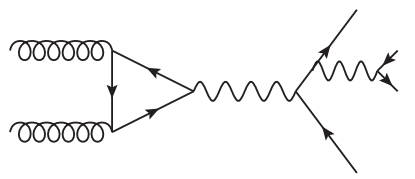

(b)



(c)

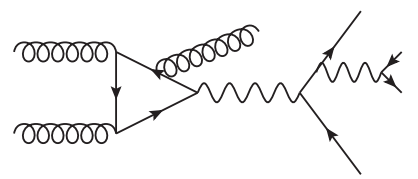

FIG. 1. Example of Feynman diagrams considered at both leading (a) and next-to-leading orders, for virtual (b) and real (c) QCD corrections.
At NLO, we need two-loop amplitudes for $g g \rightarrow$ $e^{+} e^{-} \mu^{+} \mu^{-}$and one-loop amplitudes with one extra gluon in the final state. Representative Feynman diagrams are shown in Figs. 1(b) and 1(c), respectively. The two-loop amplitudes were recently computed in Refs. [36,37] for internal massless quarks. For this study we used the implementation of these amplitudes in the GGVVAMPC++ package [38]. For the real-emission amplitudes, we implemented the result computed in Ref. [30], which provides fast and stable predictions, including in the soft and collinear regions. At variance with the results presented in Ref. [30], in this work we have also included the real radiation contributions of the form depicted on the right in Fig. 1(c). Diagrams of this kind provide the only contribution to single-resonant production, since the triangle diagrams shown on the right of Fig. 1(b) are neglected, as previously discussed. Therefore, the inclusion of the aforementioned Fig. 1(c) diagrams allows us to extend our predictions to the single-resonant region around the $Z$ boson peak.

For the real radiation contributions, the massless quark approximation we are working in holds only for $p_{4 \ell}^{T} \lesssim m_{\text {top }}$. We stress that the contributions to the total cross section outside this region are small and furthermore that our calculation only has LO accuracy for $p_{4 \ell}^{T} \neq 0$. For more reliable predictions in the high- $p_{T}$ tail, an approach based on matrix-element corrections, beyond the merging of the 0 - and the 1-jet samples already presented in [39], should be more suitable. Here, we will not focus on this aspect and leave these developments for a separate investigation.

Finally, we observe that formally the NLO corrections to $g g \rightarrow Z Z \rightarrow e^{+} e^{-} \mu^{+} \mu^{-}$include real-emission contributions of the type $q g \rightarrow Z Z \rightarrow e^{+} e^{-} \mu^{+} \mu^{-} q, g q \rightarrow Z Z \rightarrow$ $e^{+} e^{-} \mu^{+} \mu^{-} q$ and $q \bar{q} \rightarrow Z Z \rightarrow e^{+} e^{-} \mu^{+} \mu^{-} g$. We note that to include quark-initiated channels in a complete fashion, it is not sufficient to only consider the one-loop squared contributions illustrated in Figs. 2(a) and 2(c), but the full interferences contributing to the $\mathrm{N}^{3} \mathrm{LO}$ corrections to the quark-antiquark-initiated channel must be taken into account. Examples of such diagrams are illustrated in Figs. 2(b) and 2(d). They require two-loop amplitudes which are well beyond current technology.

Only including diagrams mediated by a closed fermion loop, e.g. Fig. 2(a), is possible, as they are separately gauge invariant. However, we decided not to include these contributions in our NLO calculation. Indeed, it is not clear whether they are dominant over the missing ones and whether sizable cancellations among them could in principle take place for NLO observables.

Moreover, as is well known from studies concerning Higgs-boson production, the $q g$-initiated contributions are small in the invariant mass ranges under study here and for observables which are inclusive over $p_{Z Z}^{T}$. Omitting these terms leads to an incomplete compensation of factorization scale logarithms, parametrically suppressed by the gluon/ quark luminosity ratio, that should give an indication of the size of the missing channels. 

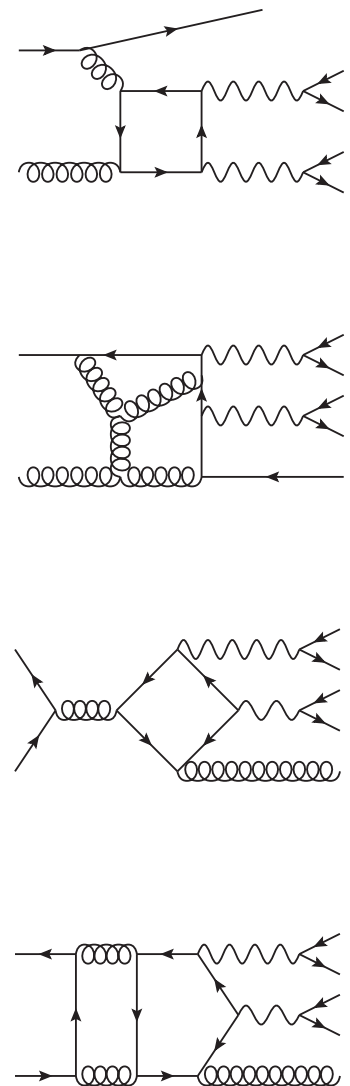

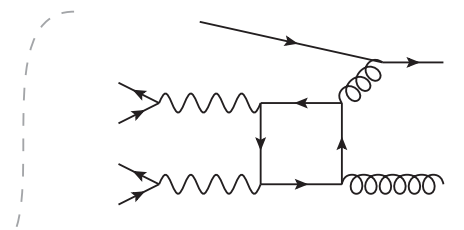

(a)

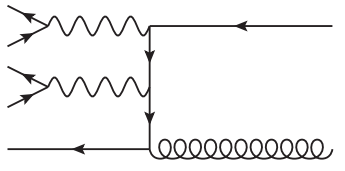

(b)

(c)
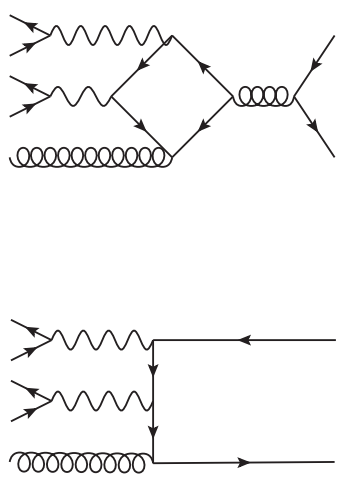

(d)

Several checks of our implementation were performed against the code used in [30], both at the level of the amplitude for single phase-space points, and at the level of integrated cross sections with static and dynamical scales. Single phase-space points were also checked against GoSam [41,42], using Ninja [43,44], and OpenLoops [45]. Furthermore, several checks on the numerical stability were performed and a rescue system that triggers the reevaluation of the unstable phase-space points in quadruple precision has been set up. Nonetheless, a technical cut $p_{Z Z}^{T}>0.5 \mathrm{GeV}$ has to be imposed to avoid instabilities in the one-loop matrix elements. By varying the cut value, we have checked that the neglected power-suppressed contributions do not significantly change the total cross section.

\section{RESULTS}

In this section we present results at LO, NLO and after interfacing with the PYTHIA8 [46] parton shower. We also include results at the so-called Les Houches event (LHE) level, i.e. after the first hard emission generated with the POWHEG method.

We consider center-of-mass energies of 8 and $13 \mathrm{TeV}$, and consider two choices of the renormalization and factorization scales

$$
\mu=\mu_{R}=\mu_{F}=m_{\mathrm{Z}}
$$

and

$$
\mu=\mu_{R}=\mu_{F}=\frac{m_{4 \ell}}{2},
$$

where

$$
m_{4 \ell}^{2}=\left(p_{e^{+}}+p_{e^{-}}+p_{\mu^{+}}+p_{\mu^{-}}\right)^{2} .
$$

For all the cases (both at LO and at NLO) we use the partonic luminosities and strong coupling from the NNPDF30_NLO_AS_0118 set [47] and fix the electroweak parameters to the following values:

$$
\begin{aligned}
m_{\mathrm{Z}}=91.1876 \mathrm{GeV} ; & \Gamma_{\mathrm{Z}}=2.4952 \mathrm{GeV} \\
m_{\mathrm{W}}=80.3980 \mathrm{GeV} ; & \sin \theta_{w}=0.2226 \\
\alpha^{-1}=132.3384 &
\end{aligned}
$$

Jets are reconstructed with the anti- $k_{\mathrm{T}}$ algorithm [48] as implemented in the FASTJET package [49,50], with jet radius $R=0.4$. Furthermore, the following kinematical cuts are applied:

$$
\begin{aligned}
& 5 \mathrm{GeV}<m_{\ell \ell}<180 \mathrm{GeV}, \\
& 60 \mathrm{GeV}<m_{4 \ell}<360 \mathrm{GeV} .
\end{aligned}
$$


The $m_{\ell \ell}$ cut is only applied to lepton pairs of the same flavor. Since we only consider the $e^{+} e^{-} \mu^{+} \mu^{-}$final state, this removes any ambiguity.

\section{A. Fixed order}

In Table I we summarize the total cross sections obtained for the setup just presented. The theoretical errors reported are due to scale variations only. We estimate them by independently varying the renormalization and factorization scales by a factor of 2 around the reference value and excluding the two extreme values of their ratio. We then use the minimum and maximum values from the resulting seven-scale combination to assign the uncertainties. To disentangle the uncertainties related to the factorization and the renormalization scales, we present independent variations of them around the central value $\mu=m_{4 \ell} / 2$ in Table II. We observe that despite the incomplete cancellation of factorization logarithms due to the omission of the $q g$-initiated channels, the final uncertainty is dominated by renormalization scale variations.

As already observed in [30] the NLO corrections are quite large and they lead to a stabilization of the scale uncertainty, whose fractional value is roughly $15 \%$ at both 8 and $13 \mathrm{TeV}$. These fractional uncertainties are slightly larger than those previously reported in Ref. [30]. We have verified that the origin of the mismatch is due to the additional scale combinations included in the envelope of our results and that perfect agreement with the previous values is obtained when we only consider 3-point variations with equal renormalization and factorization scales, as done by the authors of Ref. [30].

As expected, the two central scale values chosen $\left(m_{Z}\right.$ and $m_{4 \ell} / 2$ ) give very similar results. In the following, we use

TABLE I. $g g \rightarrow Z Z \rightarrow e^{+} e^{-} \mu^{+} \mu^{-}$total NLO cross sections and theoretical uncertainties stemming from scale variations for 8 and $13 \mathrm{TeV}$. Results for both a fixed and a dynamical choice of renormalization and factorization scales are shown.

\begin{tabular}{cccccc}
\hline \hline$[\mathrm{fb}]$ & \multicolumn{2}{c}{$\mu=m_{4 \ell} / 2$} & & \multicolumn{2}{c}{$\mu=m_{\mathrm{Z}}$} \\
\cline { 1 - 3 } \cline { 5 - 6 } $\mathrm{CME}$ & LO & NLO & & LO & NLO \\
\hline $8 \mathrm{TeV}$ & $1.60_{-0.30}^{+0.41}$ & $2.98_{-0.41}^{+0.51}$ & & $1.62_{-0.31}^{+0.42}$ & $2.98_{-0.40}^{+0.29}$ \\
$13 \mathrm{TeV}$ & $3.85_{-0.70}^{+0.97}$ & $6.98_{-0.94}^{+1.14}$ & & $3.94_{-0.71}^{+0.98}$ & $7.22_{-1.04}^{+1.04}$ \\
\hline \hline
\end{tabular}

TABLE II. $g g \rightarrow Z Z \rightarrow e^{+} e^{-} \mu^{+} \mu^{-}$total NLO cross sections and impact of separate factorization or renormalization scale variations by factors of 0.5 and 2 around the central scale $\mu=$ $m_{4 \ell} / 2$ for 8 and $13 \mathrm{TeV}$.

\begin{tabular}{lcc}
\hline \hline$[\mathrm{fb}]$ & $\mu_{R}$ fixed, $\mu_{F}$ varied & $\mu_{R}$ varied, $\mu_{F}$ fixed \\
\hline $8 \mathrm{TeV}$ & $2.98_{-0.19}^{+0.18}$ & $2.98_{-0.41}^{+0.51}$ \\
$13 \mathrm{TeV}$ & $6.98_{-0.55}^{+0.54}$ & $6.98_{-0.94}^{+1.14}$ \\
\hline \hline
\end{tabular}

$m_{4 \ell} / 2$ as our default, which is more suited over a wider range of invariant masses.

We now turn our attention to more differential observables, presenting results for the $\mathrm{LHC}$ at $13 \mathrm{TeV}$. We start by comparing the LO and NLO curves of the four-lepton invariant mass distribution $m_{4 \ell}$ in Fig. 3. Together with the main distribution, we show the differential $K$-factor of the NLO predictions divided by the LO ones in the lower inset. The lighter (darker) bands represent the 7-point (3-point) scale variation uncertainty, and in the ratio plot we also display the statistical uncertainty in form of an error bar. Both the LO and the NLO curves feature the typical enhancements due to the photon propagator contribution at low values of $m_{4 \ell}$, and the steep increase at $m_{4 \ell} \approx$ $180 \mathrm{GeV}$ due to the $Z Z$ double-resonant contribution. At NLO the single-resonant channel opens up leading to the peak at $m_{4 \ell} \approx 90 \mathrm{GeV}$. Over the rest of the spectrum the differential $K$-factor stays roughly constant at around 1.8 . This results in a flat $K$-factor for several inclusive

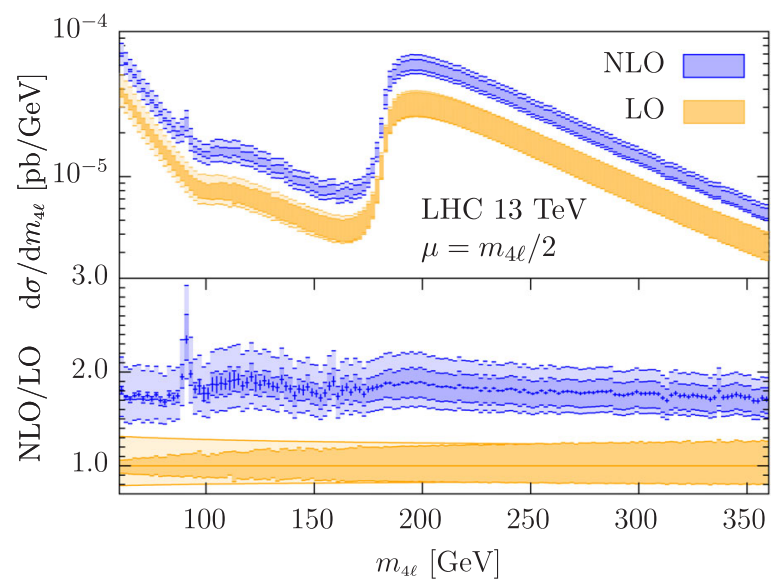

FIG. 3. Four-lepton invariant mass $m_{4 \ell}$ distribution at LO and NLO for the LHC at $13 \mathrm{TeV}$.

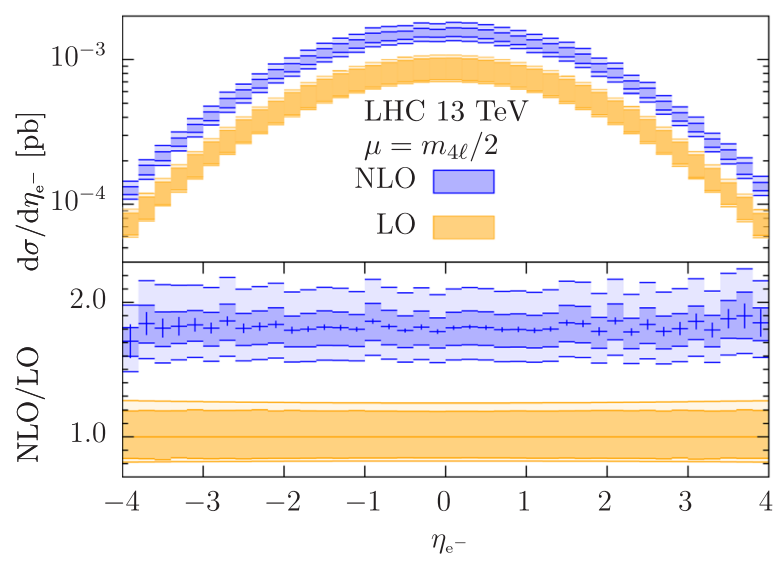

FIG. 4. Electron pseudorapidity distribution at LO and NLO for the $\mathrm{LHC}$ at $13 \mathrm{TeV}$. 




FIG. 5. Azimuthal separation between electron and muon at LO and NLO for the LHC at $13 \mathrm{TeV}$.

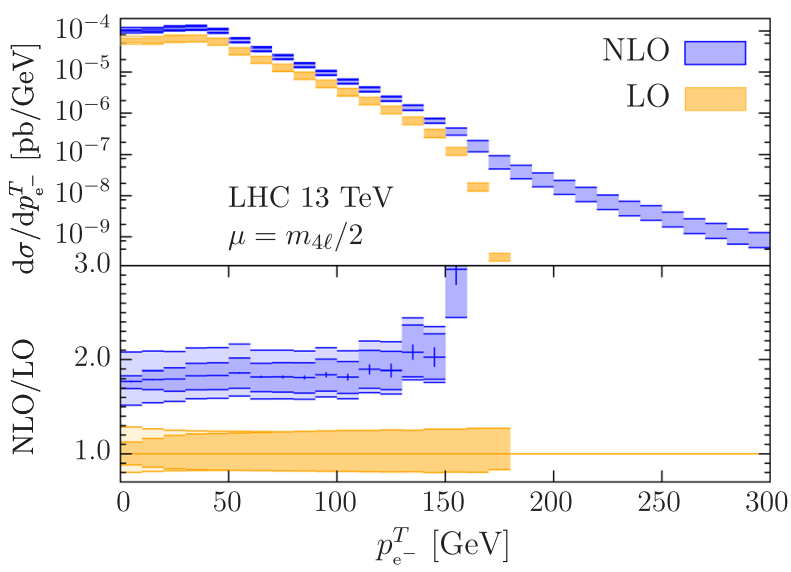

FIG. 6. Electron transverse momentum distribution at LO and NLO for the LHC at $13 \mathrm{TeV}$.

distributions. As an example, we show in Fig. 4 the pseudorapidity of the electron.

Next, in Fig. 5 we investigate the azimuthal separation between $e^{-}$and $\mu^{-}$, which provides interesting information about the diboson production mechanism [51]. A constant $K$-factor is again observed across almost the whole spectrum, with small deviations only visible in the region around 180 degrees.

The situation is different for observables which are sensitive to extra QCD radiation. An interesting example is the transverse momentum distribution of the electron, shown in Fig. 6. At leading order the curve has an upper kinematical bound at $p_{\mathrm{e}^{-}}^{T}=180 \mathrm{GeV}$ due to the upper limit on $m_{4 \ell}$ introduced in Eq. (5). In the low end of the spectrum this observable is predicted at NLO accuracy and it shows a flat $K$-factor. The additional radiation from the real-emission contribution allows the electrons to be produced with a transverse momentum larger than the kinematic limit of $180 \mathrm{GeV}$. This means that, above this value, the NLO curve effectively becomes LO. This is reflected in the population of this region and in the enlarged scale uncertainty band.

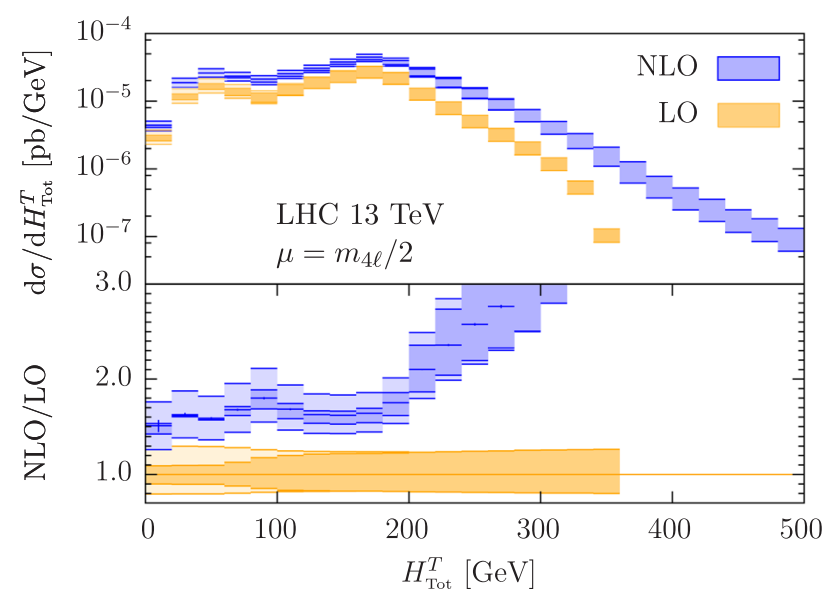

FIG. 7. $H_{\mathrm{Tot}}^{T}$ distribution at LO and NLO for the LHC at $13 \mathrm{TeV}$.

A similar sensitivity to a kinematic threshold is also present in the differential distribution of $H_{\text {Tot }}^{T}$ shown in Fig. 7 and defined as

$$
H_{\mathrm{Tot}}^{T}=p_{e^{+}}^{T}+p_{e^{-}}^{T}+p_{\mu^{+}}^{T}+p_{\mu^{-}}^{T}+\sum_{j \in \mathcal{J}} p_{j}^{T},
$$

where the sum runs over the set $\mathcal{J}$ of final state jets. At fixed NLO there can of course be at most one resolved jet, due to the real radiation emission. The situation can however be more involved after the shower, and we will comment further on this in the next section.

\section{B. Fixed order vs POWHEG first emission}

As a next step, we compare predictions at the NLO and at the LHE level, meaning with the addition of the first hard emission generated according to the POWHEG method. The results at the LHE level are unphysical, but the comparison with the fixed-order results and, later on, with the fully showered ones helps in assessing how big are the effects due to the exponentiation intrinsic in the POWHEG method and in separating them from the pure showering.

In order to avoid an excessive enhancement of the hightransverse momentum tail of the $Z Z$ pair and of the hardest jet, previously observed in similar POWHEG implementations of processes with large $K$-factors and discussed at length in Refs. [52,53], we have chosen to limit the amount of real radiation that gets exponentiated by the Sudakov factor by setting the HDAMP $[52,53]$ parameter in the POWHEG BOX to $100 \mathrm{GeV}$. This effectively ensures that we smoothly recover the exact NLO result above that scale.

Figure 8 shows again the four-lepton invariant mass spectrum at NLO and LHE level. Apart from some statistical fluctuations caused by the narrow binning, which we kept in order to highlight the single-resonant peak at $m_{4 \ell}=m_{\mathrm{Z}}$, the agreement between LHE and NLO predictions is good over the whole kinematical range. This is the expected result for observables which are inclusive over 


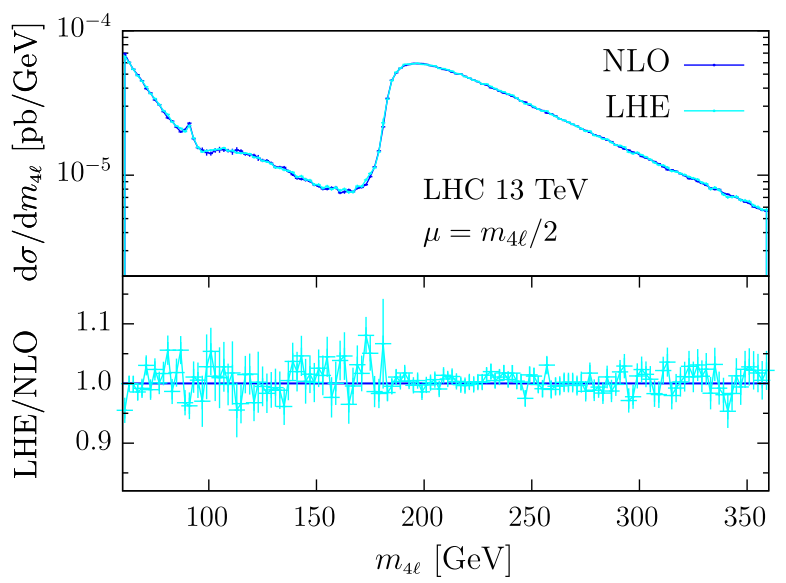

FIG. 8. Invariant mass distribution of the four-lepton system at NLO and LHE level at $13 \mathrm{TeV}$.

the extra radiation generated by POWHEG. We have verified that similar results are obtained for several other inclusive observables, e.g. the rapidities of the leptons, or of the reconstructed $Z$ bosons.

The situation is different when we consider the transverse momentum of the four-lepton system, shown in Fig. 9. Since the four-lepton system recoils against the real radiation emission and has vanishing transverse momentum when the emission becomes soft or collinear, this observable is directly sensitive to the real radiation. The NLO curve diverges for $p_{4 \ell}^{T} \rightarrow 0$. However, when the real radiation is weighted by the Sudakov form factor in the LHE-level predictions, we observe the effect of the Sudakov suppression and the distribution becomes finite for vanishing transverse momenta. Far away from the Sudakov region, for transverse momenta $p_{4 \ell}^{T}>150 \mathrm{GeV}$ the NLO and LHE gets closer, as expected following the usage of the HDAMP factor in the POWHEG implementation.

We note, however, that even after the inclusion of the HDAMP factor an exact agreement between the NLO and

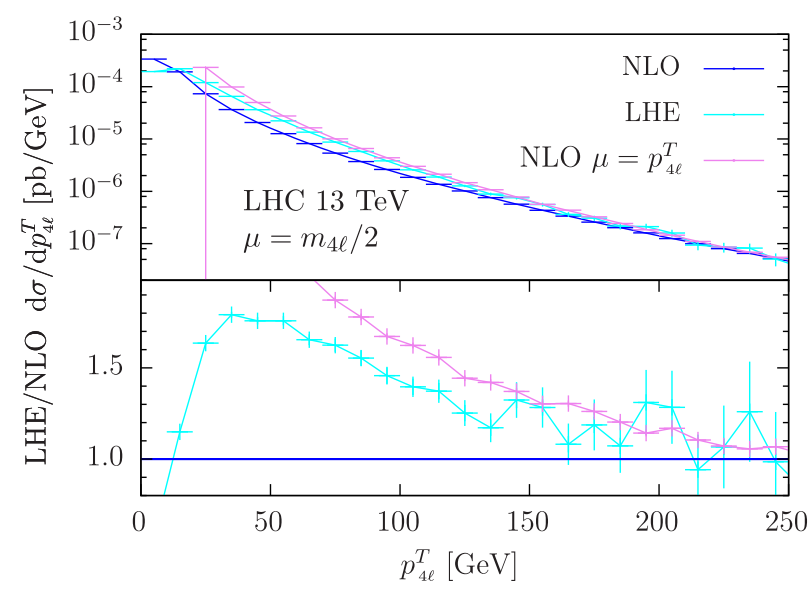

FIG. 9. Transverse momentum distribution of the four-lepton system at NLO and LHE level at $13 \mathrm{TeV}$.
LHE results in the tail of the distribution should not be expected. This is a consequence of the different choices for the renormalization and factorization scales used in the two calculations. The matrix element for the real emission in the POWHEG method has the strong coupling constant associated with the extra emission evaluated at $\mu_{R}=\mu_{F}=p_{4 \ell}^{T}$. The NLO result is instead evaluated at $\mu_{R}=\mu_{F}=m_{4 \ell} / 2$. Since the usage of the HDAMP parameter provides a smooth interpolation - rather than a hard switch-between the two, the final combination will not be identical to the NLO result.

In order to quantify the effects of this discrepancy in Fig. 9 we also plot the fixed-order results above $p_{4 l}^{T}>$ $20 \mathrm{GeV}$ choosing $\mu_{R}=\mu_{F}=p_{4 \ell}^{T}$ for all the powers of $\alpha_{s}$. We see that a reasonable agreement between the three curves is reached above $200-250 \mathrm{GeV}$, before the two NLO curves start to depart for higher values of the scales (not shown in the plot). In any case, we would like to stress that due to the massless-quark approximation we are working in, the predictions for $p_{4 \ell}^{T}$ (or correspondingly $p_{j}^{T}$ ) should not be trusted for larger values of the transverse momentum, because the effects of the massive top quarks in the loop can no longer be neglected.

\section{Showered results}

We now turn to the study of the impact of the parton shower. The results showed in the following are produced using PYTHIA8 for the showering and hadronization stages. In order to keep the analysis simpler and to have a more direct comparison with theoretical predictions at the partonic level, we have decided not to include multiple parton interactions in the following plots.

We also remark that the limitation to only consider the gluon-initiated channel that is used at the fixed order or LHE level is removed when we interface with the parton shower, which is free to generate $q \rightarrow q g$ initial-state splittings. This is allowed by the unitary of the backward-evolved parton shower, which for a given hard process produces the same total cross section irrespective of the partonic splittings allowed. To quantify the impact of the inclusion of the quarks in the shower, we have also studied the extreme case where the shower is only allowed to perform $g \rightarrow g g$ splittings. ${ }^{3}$ No appreciable differences for differential distributions are found, apart from two expected exceptions. First, the transverse momentum of the hardest jet at very low values, which is clearly affected by the number and type of splittings included in the Sudakov exponent. Second, the inclusion of quarks leads to mildly harder transverse-momentum spectra, as already observed

\footnotetext{
${ }^{3}$ This can be achieved by setting SPACESHOWER:NQUAR$\mathrm{KIN}=0$ SpaceShower $:$ nQuarkIn $=0$ in PYTHIA8. Note that this removes quarks altogether, which is different from our large gluon flux approximation. As such, this only provides an upper bound on effects due to the presence of quarks in the shower.
} 




FIG. 10. Invariant mass distribution of the four-lepton system at the LHE level and after shower and hadronization with PYTHIA8, compared to the fixed NLO curve.

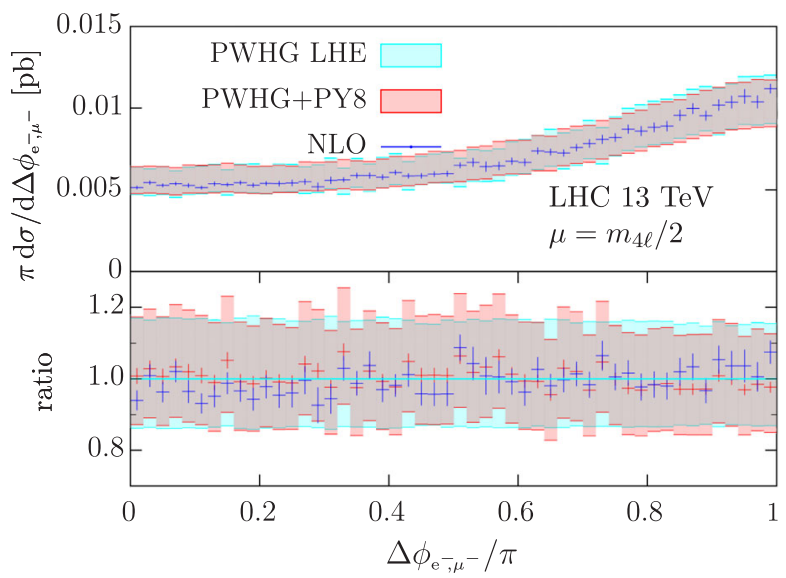

FIG. 11. Same as Fig. 10 but for the azimuthal separation between the electron and the muon.

in [39]. Thus, there are no major inconsistencies in allowing $q \rightarrow q g$ splitting in the parton shower despite $q g$-initiated channels having been omitted in the hard matrix element. However, we emphasize that the former is not a substitute for the latter, nor can this be taken as an indication that the omitted $q g$-initiated channels are small.

In Figs. 10-15 we compare the showered results to the NLO and LHE results at the nominal scale $\mu=m_{4 \ell} / 2$. In all the observables we note a scale uncertainty which varies around $20 \%$, as is the case for fixed-order predictions.

For observables which are inclusive over the extra radiation, we note an excellent agreement between the LHE-level results and the POWHEG+PYTHIA8 predictions. This is also true for the theory uncertainty bands which overlap almost perfectly. As one would expect, the parton shower does not have a strong influence on these quantities. This is shown for the four-lepton invariant mass distribution, in Fig. 10, in which the single-resonant peak is still clearly visible. We have verified that the shower has a similarly small effect for several other inclusive

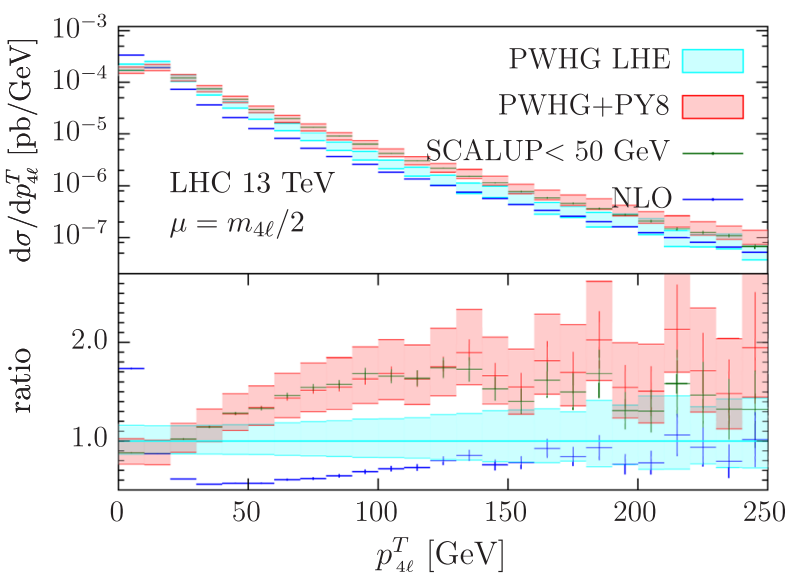

FIG. 12. Same as Fig. 10 but for the transverse momentum of the four-lepton system.

distributions, including the rapidities of the leptons and of the $Z$ bosons. As a further example, we show the azimuthal separation between $e^{-}$and $\mu^{-}$in Fig. 11.

By contrast, the parton shower has a larger impact on the transverse momentum of the four lepton system, Fig. 12. At small transverse momenta it undershoots the LHE-level prediction by roughly $10 \%$, but above $30 \mathrm{GeV}$ it becomes larger than the LHE-level results, reaching a plateau around $150 \mathrm{GeV}$, where the ratio between the two predictions is between 1.5 and 2. The large discrepancy between the showered results and the fixed-order (or the LHE level) ones in the tail of the distribution, which are still however roughly compatible given the correspondingly large LO scale variations, can be explained by the fact that by adding further radiation the shower increases the transverse momentum of the color-neutral four-lepton system, which has to recoil against the sum of all emitted particles. This can be further demonstrated by lowering the starting scale for the PYTHIA8 showering: for example, in Fig. 12 we also include predictions where we have limited the hardness of shower emissions to be lower than $50 \mathrm{GeV}$, irrespective of the hardness of the first POWHEG emission. ${ }^{4}$ The resulting predictions in the large $p_{4 \ell}^{T}$ region are closer to the NLO curve, due to the reduced PYTHIA8 activity. The same effect is seen in Fig. 13 for the scalar sum of the transverse momenta defined in Eq. (6). We would like to stress that such a large effect caused by the shower in this region has to be taken with great care since it comes with a very large uncertainty, as illustrated by the SCALUP variation. However, we remind the reader that an accurate description of the high- $p_{4 \ell}^{T}$ region is not within the scope of this paper and is intrinsically problematic within our approximation. Currently, in order to obtain a more reliable description of this region one could merge leading order

\footnotetext{
${ }^{4}$ This is done by limiting the SCALUP value to $50 \mathrm{GeV}$.
} 


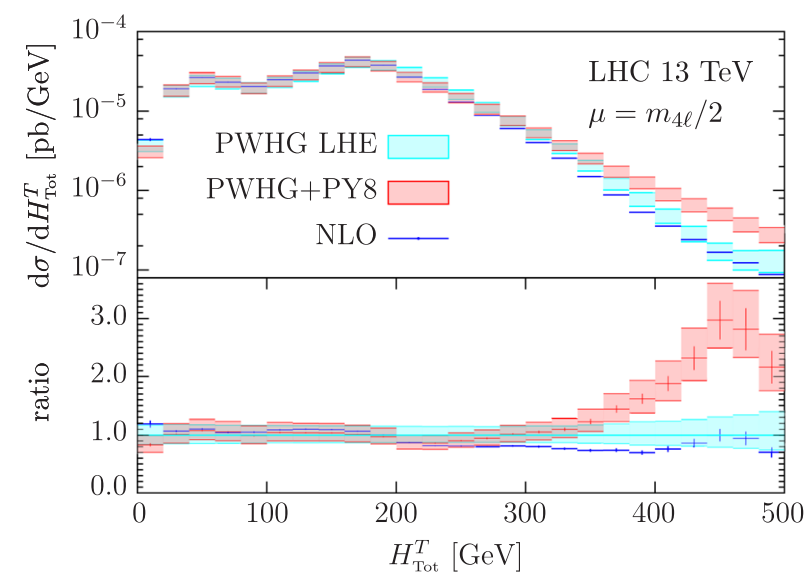

FIG. 13. Same as Fig. 10 but for $H_{\text {Tot }}^{T}$, with jets reconstructed by the anti- $k_{T}$ algorithm with $R=0.4$.

samples-including quark-initiated processes and full mass dependence - with at least 2 jets.

On the contrary, a similar enhancement is not expected when looking at the transverse momentum of the hardest jet in the event. Indeed, the shower emissions are by construction subdominant with respect to the leading jet and on average are separated enough not to be clustered with it. Therefore, while the shower has a larger effect on the transverse momentum of the colorless recoiling system, it should not significantly affect the leading-jet spectrum. This is observed in Fig. 14, which only displays a mild softening of the leading jet $p_{T}$ with respect to the LHE results, due to radiation off the jet.

In our analysis the two $Z$ bosons are reconstructed according to their invariant mass. Event by event we distinguish two $Z$ bosons: the one whose invariant mass is closer to $m_{Z}$, labeled $Z_{1}$, and the one further away, labeled $Z_{2}$. Since we consider the $e^{+} e^{-} \mu^{+} \mu^{-}$final state, $Z_{1}$ and $Z_{2}$ are always reconstructed by opposite sign leptons from the same family. However, this procedure allows to

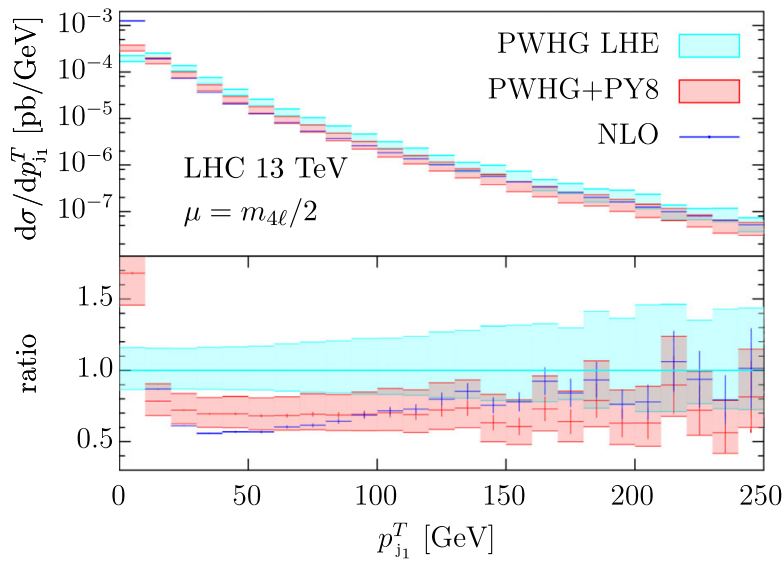

FIG. 14. Same as Fig. 10 but for the transverse momentum of the hardest jet, reconstructed by the anti- $k_{T}$ algorithm with $R=0.4$.

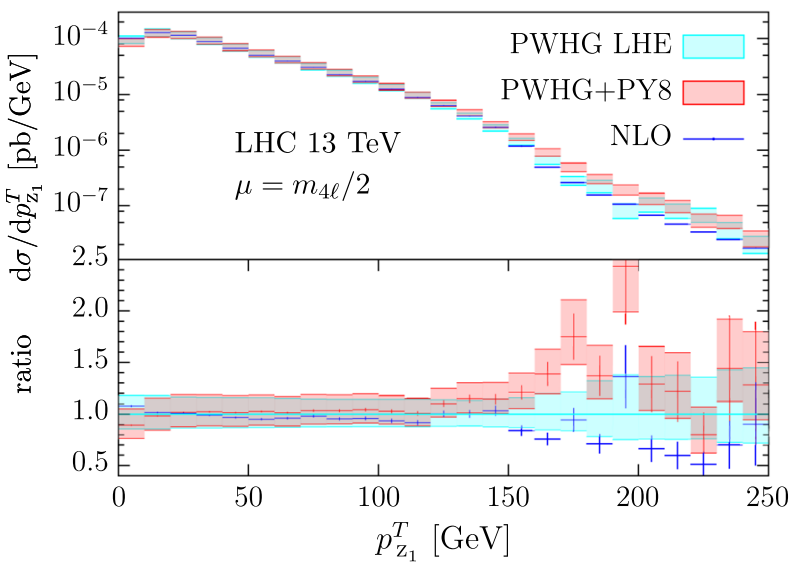

FIG. 15. Same as Fig. 10 but for the transverse momentum of the $Z$ boson whose invariant mass is closer to the mass peak.

uniquely define the two $Z$ bosons also for final states with equal pairs of leptons.

The transverse momentum of the $Z_{1}$ boson, displayed in Fig. 15, is almost unaffected by parton shower corrections for values of the transverse momentum smaller than $150 \mathrm{GeV}$. For harder values of $p_{Z_{1}}^{T}$ the shower increases the cross section. This effect is related to the crossing of the kinematic threshold already observed in the comparison of LO and NLO predictions for the electron transverse momentum in Fig. 6.

\section{ATLAS fiducial cuts}

Before concluding we present results obtained applying fiducial cuts similar to the ones used by the ATLAS Collaboration in [54], namely:

$$
\begin{aligned}
80 \mathrm{GeV} & <m_{4 \ell}<350 \mathrm{GeV}, \\
66 \mathrm{GeV} & <m_{\ell \ell}<160 \mathrm{GeV}, \\
\Delta R_{\ell \ell} & >0.2, \\
p_{\ell}^{T} & >7 \mathrm{GeV}, \\
\left|\eta_{\ell}\right| & >2.7 .
\end{aligned}
$$

Within these fiducial cuts, the resulting NLO cross section at $13 \mathrm{TeV}$ is

$$
\sigma^{\mathrm{fid}}=4.57_{-0.59}^{+0.71} \mathrm{fb} .
$$

In Fig. 16-20 we compare the corresponding POWHEG +PYTHIA8 predictions with pure NLO ones for the fourlepton invariant mass and transverse momentum, for the transverse momentum of the $Z_{1}$ boson and for the pseudorapidity and transverse momentum of the electron, respectively. In the lower inset of each plot we show the scale uncertainty band obtained with a 7-point variation of renormalization and factorization scales, as explained in the previous section. The same features observed for the more 


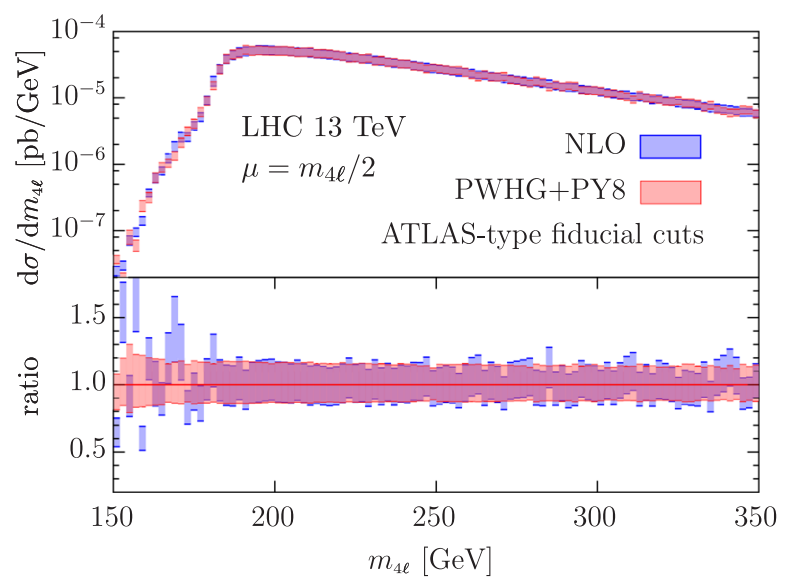

FIG. 16. Invariant mass distribution of the four-lepton system at NLO and after shower and hadronization with PYTHIA8 when ATLAS fiducial cuts are applied.

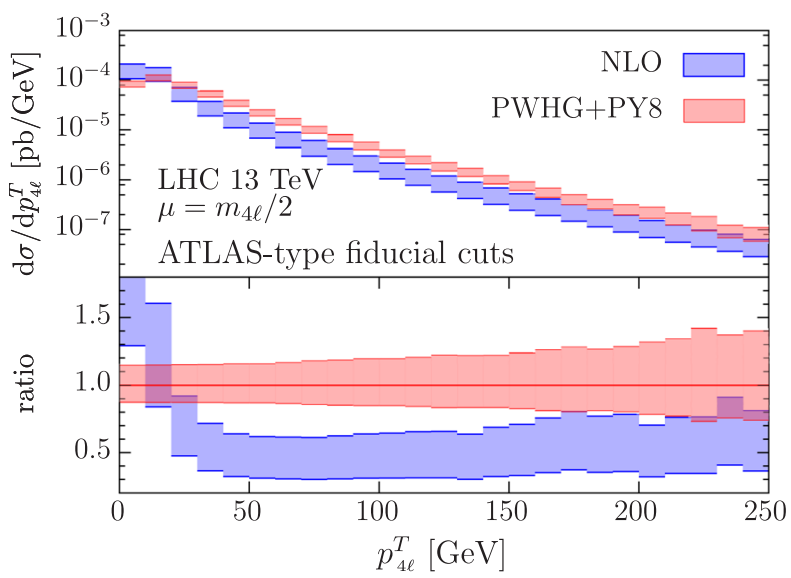

FIG. 17. Transverse momentum distribution of the four-lepton system at NLO and after shower and hadronization with PYTHIA8 when ATLAS fiducial cuts are applied.

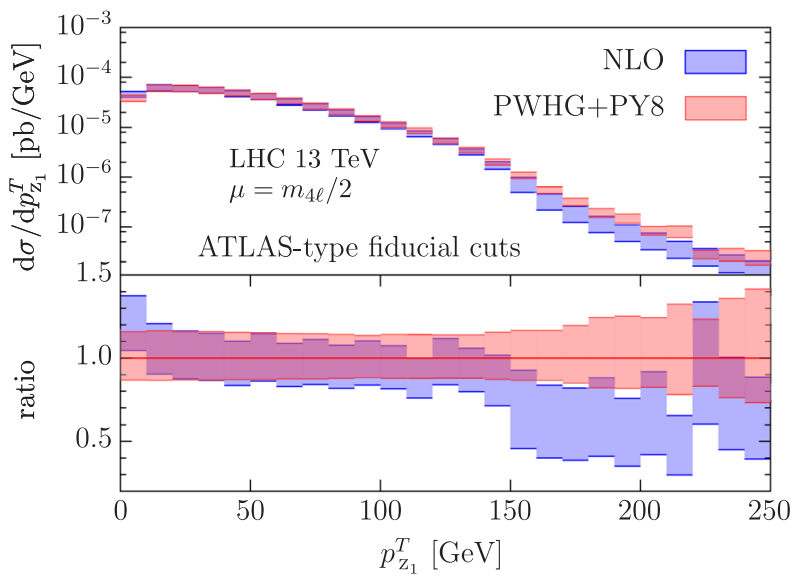

FIG. 18. Transverse momentum distribution of the $Z$ boson, whose invariant mass is closer to the mass peak, at NLO and after shower and hadronization with PYTHIA8 when ATLAS fiducial cuts are applied.

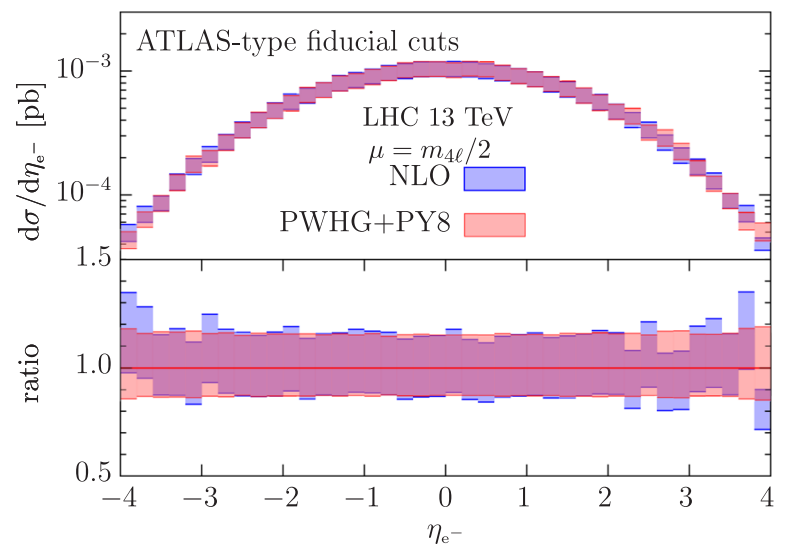

FIG. 19. Rapidity distribution of the electron at NLO and after shower and hadronization with PYTHIA8 when ATLAS fiducial cuts are applied.

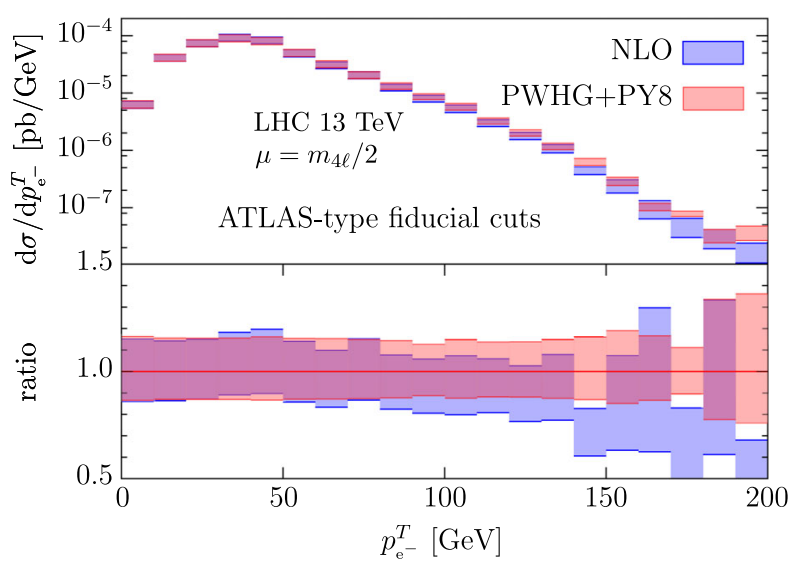

FIG. 20. Transverse momentum distribution of the electron at NLO and after shower and hadronization with PYTHIA8 when ATLAS fiducial cuts are applied.

inclusive analysis are present in this fiducial region, as expected for such inclusive cuts.

\section{CONCLUSIONS AND OUTLOOK}

The production of a pair of $Z$ bosons plays a key role at the LHC, not only as a further important test of the Standard Model, but in relation to Higgs studies. In this paper we have interfaced a NLO computation for $Z Z$ production in gluon fusion to a parton shower, using the POWHEG BOX framework. The calculation has been performed in the limit of the gluon PDF being much larger than any quark one. We also neglected quark mass effects in the loops throughout. In this study we have primarily concentrated on the $Z Z$-production process as a signal and have consequently not attempted to include either the Higgs-boson mediated channel or the interference between the two production modes. These effects are known to be important and severely affect the production rates mostly 
around the Higgs-boson resonant region and for large values of $m_{4 \ell}$ [32-34]. The proper inclusion of all the aforementioned effects in a NLO calculation matched to parton shower will be the subject of a separate investigation.

After interfacing with the parton shower in PYTHIA8, our findings are in agreement with the expectations. In particular, we observe that quantities which are inclusive over the extra radiation do not receive appreciable modifications by the showering stage. On the other hand, there is a substantial effect due to the parton shower for quantities that are more sensitive to the hadronic activity, even when the observables are built exclusively using the four momenta of the leptons coming from the $Z$ decays. A typical example is the transverse momentum of the fourlepton system, which at NLO is constrained by momentum conservation to recoil against the emitted parton. Other situations where the parton shower provides large corrections appear in the presence of multiple shower emissions, which allow observables to evade kinematical bounds that would be otherwise present at fixed-order. This is e.g. observed in the transverse momentum of hardest $Z$ boson, above the kinematical bound set by the generation cuts on $m_{4 \ell}$. We have found similar effects also when applying realistic experimental cuts, modeled on those used by the ATLAS Collaboration in a previous analysis of the $Z Z$ four-lepton final state. We provided predictions in this fiducial region for the $13 \mathrm{TeV}$ LHC Run II.

There are several interesting further developments we did not investigate in this first study: for example the inclusion of quark-mass effects in the loops as well as a detailed study of the Higgs-mediated contributions and their relevance for off-shell Higgs analyses. Also, it would be interesting to explore the impact of the matching to different parton showers and their comparison with matrixelements corrected approaches. We leave these for future investigations.

\section{ACKNOWLEDGMENTS}

We thank J. Lindert for help and support in comparing the analytic amplitudes used in this calculation with OpenLoops. We also thank E. Re and P. Nason for useful conversations and suggestions about the optimal usage of new features of the POWHEG BOX v2. The numerical computations were performed at the RZG-the Rechenzentrum Garching near Munich Garching. This work was supported by the COFUND Fellowship under Grant Agreement No. PCOFUND-GA-2012-600377 (S. A.), and by the German Federal Ministry for Education and Research (BMBF) under Grant No. 05H15VKCCA (R. R.).
[1] G. Aad et al. (ATLAS Collaboration), Phys. Rev. Lett. 112, 231806 (2014).

[2] G. Aad et al. (ATLAS Collaboration), Phys. Lett. B 753, 552 (2016).

[3] CMS Collaboration, Report No. CMS-PAS-SMP-15-012, 2016.

[4] G. Aad et al. (ATLAS Collaboration), J. High Energy Phys. 09 (2016) 029.

[5] V. Khachatryan et al. (CMS Collaboration), Phys. Lett. B 763, 280 (2016).

[6] V. Khachatryan et al. (CMS Collaboration), Eur. Phys. J. C 76, 401 (2016).

[7] G. Aad et al. (ATLAS Collaboration), Phys. Lett. B 716, 1 (2012).

[8] S. Chatrchyan et al. (CMS Collaboration), Phys. Lett. B 716, 30 (2012).

[9] V. Khachatryan et al. (CMS Collaboration), J. High Energy Phys. 04 (2016) 005.

[10] G. Aad et al. (ATLAS Collaboration), Phys. Rev. Lett. 115, 091801 (2015).

[11] G. Aad et al. (ATLAS Collaboration), Phys. Rev. Lett. 116, 101801 (2016).

[12] S. Chatrchyan et al. (CMS Collaboration), J. High Energy Phys. 01 (2013) 063.

[13] V. Khachatryan et al. (CMS Collaboration), Phys. Lett. B 740, 250 (2015); 757, 569(E) (2016).
[14] G. Aad et al. (ATLAS Collaboration), J. High Energy Phys. 03 (2013) 128.

[15] M. Grazzini, S. Kallweit, S. Pozzorini, D. Rathlev, and M. Wiesemann, J. High Energy Phys. 08 (2016) 140.

[16] T. Gehrmann, M. Grazzini, S. Kallweit, P. Maierhöfer, A. von Manteuffel, S. Pozzorini, D. Rathlev, and L. Tancredi, Phys. Rev. Lett. 113, 212001 (2014).

[17] M. Grazzini, S. Kallweit, D. Rathlev, and M. Wiesemann, Phys. Lett. B 761, 179 (2016).

[18] M. Grazzini, S. Kallweit, and D. Rathlev, arXiv:1601.06751.

[19] M. Grazzini, S. Kallweit, and D. Rathlev, J. High Energy Phys. 07 (2015) 085.

[20] M. Grazzini, S. Kallweit, D. Rathlev, and A. Torre, Phys. Lett. B 731, 204 (2014).

[21] M. Grazzini, S. Kallweit, and D. Rathlev, Phys. Lett. B 750, 407 (2015).

[22] F. Cascioli, T. Gehrmann, M. Grazzini, S. Kallweit, P. Maierhöfer, A. von Manteuffel, S. Pozzorini, D. Rathlev, L. Tancredi, and E. Weihs, Phys. Lett. B 735, 311 (2014).

[23] S. Catani, L. Cieri, D. de Florian, G. Ferrera, and M. Grazzini, Phys. Rev. Lett. 108, 072001 (2012); 117, 089901 (E) (2016).

[24] J. M. Campbell, R. K. Ellis, Y. Li, and C. Williams, J. High Energy Phys. 07 (2016) 148.

[25] M. Grazzini, S. Kallweit, D. Rathlev, and M. Wiesemann, J. High Energy Phys. 08 (2015) 154. 
[26] S. Dawson, P. Jaiswal, Y. Li, H. Ramani, and M. Zeng, Phys. Rev. D 94, 114014 (2016).

[27] T. Binoth, M. Ciccolini, N. Kauer, and M. Kramer, J. High Energy Phys. 03 (2005) 065.

[28] T. Binoth, M. Ciccolini, N. Kauer, and M. Kramer, J. High Energy Phys. 12 (2006) 046.

[29] T. Binoth, N. Kauer, and P. Mertsch, arXiv:0807.0024.

[30] F. Caola, K. Melnikov, R. Röntsch, and L. Tancredi, Phys. Rev. D 92, 094028 (2015).

[31] F. Caola, K. Melnikov, R. Röntsch, and L. Tancredi, Phys. Lett. B 754, 275 (2016).

[32] F. Caola, M. Dowling, K. Melnikov, R. Röntsch, and L. Tancredi, J. High Energy Phys. 07 (2016) 087.

[33] J. M. Campbell, R. K. Ellis, M. Czakon, and S. Kirchner, J. High Energy Phys. 08 (2016) 011.

[34] N. Kauer and G. Passarino, J. High Energy Phys. 08 (2012) 116.

[35] http://mcfm.fnal.gov.

[36] F. Caola, J. M. Henn, K. Melnikov, A. V. Smirnov, and V. A. Smirnov, J. High Energy Phys. 06 (2015) 129.

[37] A. von Manteuffel and L. Tancredi, J. High Energy Phys. 06 (2015) 197.

[38] https://vvamp.hepforge.org/.

[39] F. Cascioli, S. Höche, F. Krauss, P. Maierhöfer, S. Pozzorini, and F. Siegert, J. High Energy Phys. 01 (2014) 046.

[40] S. Alioli, P. Nason, C. Oleari, and E. Re, J. High Energy Phys. 06 (2010) 043.
[41] G. Cullen, N. Greiner, G. Heinrich, G. Luisoni, P. Mastrolia, G. Ossola, T. Reiter, and F. Tramontano, Eur. Phys. J. C 72, 1889 (2012).

[42] G. Cullen, H. van Deurzen, N. Greiner, G. Heinrich, G. Luisoni et al., Eur. Phys. J. C 74, 3001 (2014).

[43] H. van Deurzen, G. Luisoni, P. Mastrolia, E. Mirabella, G. Ossola, and T. Peraro, J. High Energy Phys. 03 (2014) 115.

[44] T. Peraro, Comput. Phys. Commun. 185, 2771 (2014).

[45] F. Cascioli, P. Maierhofer, and S. Pozzorini, Phys. Rev. Lett. 108, 111601 (2012).

[46] T. Sjostrand, S. Mrenna, and P. Z. Skands, Comput. Phys. Commun. 178, 852 (2008).

[47] R. D. Ball et al. (NNPDF Collaboration), J. High Energy Phys. 04 (2015) 040.

[48] M. Cacciari, G. P. Salam, and G. Soyez, J. High Energy Phys. 04 (2008) 063.

[49] M. Cacciari and G. P. Salam, Phys. Lett. B 641, 57 (2006).

[50] M. Cacciari, G. P. Salam, and G. Soyez, Eur. Phys. J. C 72, 1896 (2012).

[51] S. Bolognesi, Y. Gao, A. V. Gritsan, K. Melnikov, M. Schulze, N. V. Tran, and A. Whitbeck, Phys. Rev. D 86, 095031 (2012).

[52] S. Alioli, P. Nason, C. Oleari, and E. Re, J. High Energy Phys. 04 (2009) 002.

[53] S. Alioli, P. Nason, C. Oleari, and E. Re, J. High Energy Phys. 09 (2009) 111.

[54] ATLAS Collaboration, Report No. ATLAS-CONF-2013020, 2013. 